\title{
Comment on "Hydrochlorothiazide use and the risk of skin cancer in patients with hypertensive disorder: a nationwide retrospective cohort study from Korea"
}

\author{
Anton Pottegård ${ }^{1}$ and Daniel Morales ${ }^{2}$
}

\begin{abstract}
${ }^{1}$ Clinical Pharmacology and Pharmacy, Department of Public Health, University of Southern Denmark, Odense, Denmark; ${ }^{2}$ Division of Population Health and Genomic, University of Dundee, Dundee, UK
\end{abstract}

Received: January 11, 2020 Accepted: March 1, 2020

\section{Correspondence to}

Anton Pottegård, M.D.

Clinical Pharmacology and Pharmacy, Department of Public Health, University of Southern Denmark, JB Winsløws Vej 19, 5000 Odense, Denmark

Tel: +45-65503024

E-mail: apottegaard@health.sdu.dk https://orcid.org/0000-0001-93145679
We read with interest the paper by Park et al. [1] investigating the association between hydrochlorothiazide and non-melanoma and melanoma skin cancer in South Korean patients. We were, however, surprised to read that hydrochlorothiazide use was inversely associated with skin cancer risk. This contradicts previous studies [2,3], the underlying biological rationale based on hydrochlorothiazide's known photosensitizing properties, as well as conclusions reached by medicines regulators [4]. We believe, however, that these contrasting findings arise due to bias in the study design.

In the study, Park et al. [1] follow patients from their first antihypertensive prescription which, as described, may not be a prescription for hydrochlorothiazide in the hydrochlorothiazide exposed group. Hydrochlorothiazide is often prescribed in combination with other antihypertensives and may therefore be less likely to be prescribed first-line. In the absence of a time-varying analysis, this will induce immortal time bias in the hydrochlorothiazide user group.

Patients were followed until the last day of data capture or the day of the first diagnosis of skin cancer. Exposure to hydrochlorothiazide was analyzed as having at least three hydrochlorothiazide prescriptions filled throughout that patient's total follow-up. However, the duration of follow-up is shorter for patients receiving a skin cancer diagnosis (as this censor follow-up). Having filled $\geq 50,000 \mathrm{mg}$ of hydrochlorothiazide (the highest strata in the dose-response analyses) will, with a high dose of $25 \mathrm{mg} /$ day, take $>5$ years, or with $12.5 \mathrm{mg} /$ day $>10$ years. This has to be achieved within the maximum study period of 7.5 years (January 2009 to June 2017). As such, achieving such cumulative hydrochlorothiazide exposure levels is equivalent to requiring the near-total absence of a censoring event. Such bias will be much more pronounced in the dose-response analyses and corresponds quite well to the finding that exposure yields a very strong 'protective' effect against skin cancer.

Whether these issues have been correctly identified, and whether they explain the findings or not, can easily be tested by the authors. First, any antihypertensive drug should display similar properties, that is, if the authors performs similar cumulative analyses using their design for e.g., angiotensin-converting enzyme inhibitors or calcium channel blockers, we would 
expect them to identify similar protective associations. Second, hydrochlorothiazide use should be handled as a time-varying exposure. As such, each individual should contribute to the non-hydrochlorothiazide cohort from the time of the first antihypertensive prescription until the time of the first hydrochlorothiazide prescription. From the time of the first hydrochlorothiazide prescription, that person should contribute person-time to the hydrochlorothiazide user group only. If person-time is handled in this way, we would expect the protective associations obtained for 'ever use of hydrochlorothiazide' to vanish.

Nevertheless, we may not expect to see similar risks observed in a European population [2,3] replicated in an Asian population. In fact, in a recent Taiwanese study, hydrochlorothiazide showed no significant association to skin cancer [5]. This may be explained by several factors, including differences in skin phenotype, use of lower hydrochlorothiazide doses, and cultural differences in sun behavior. However, additional studies in Asian populations are necessary to confirm these findings.

\section{Conflict of interest}

No potential conflict of interest relevant to this article was reported.

\section{REFERENCES}

1. Park E, Lee Y, Jue MS. Hydrochlorothiazide use and the risk of skin cancer in patients with hypertensive disorder: a nationwide retrospective cohort study from Korea. Korean J Intern Med 2020;35:917-928.

2. Pedersen SA, Gaist D, Schmidt SAJ, Holmich LR, Friis S, Pottegard A. Hydrochlorothiazide use and risk of nonmelanoma skin cancer: a nationwide case-control study from Denmark. J Am Acad Dermatol 2018;78:673-681.e9.

3. Morales DR, Pacurariu A, Slattery J, Kurz X. Association between hydrochlorothiazide exposure and different incident skin, lip and oral cavity cancers: A series of population-based nested case-control studies. Br J Clin Pharmacol 2020 Feb 18 [Epub]. https://doi.org/10.1111/bcp.14245.

4. European Medicines Agency. PRAC recommendations on signals [Internet]. London (UK): European Medicines Agency, 2018 [cited 2020 Mar 10]. Available from: https:// www.ema.europa.eu/en/documents/prac-recommendation/prac-recommendations-signals-adopted-3-6-september-2018-prac-meeting_en-o.pdf.

5. Pottegard A, Pedersen SA, Schmidt SAJ, et al. Use of hydrochlorothiazide and risk of skin cancer: a nationwide Taiwanese case-control study. Br J Cancer 2019;121:973-978. 